\title{
Evaluation of the incremental prognostic value of the combination of CYP2C19 poor metabolizer status and $A B C B 1$ 3435 TT polymorphism over conventional risk factors for cardiovascular events after drug-eluting stent implantation in East Asians
}

\author{
Mahn-Won Park, MD, PhD, Sung Ho Her, MD, PhD¹, Chan Joon Kim, MD¹, Jung SunCho, MD, PhD', \\ Gyung-Min Park, MD, PhD'1, Tae-Seok Kim, MD1, Yun-Seok Choi, MD, PhD², Chul-Soo Park, MD, PhD², \\ Yoon-Seok Koh, MD, PhD ${ }^{3}$, Hun-Jun Park, MD, PhD³, Pum-Joon Kim, MD, PhD, \\ Wook-Sung Chung, MD, PhD³, Ki-Bae Seung, MD, PhD ${ }^{3}$, Ho-Sook Kim, PhD ${ }^{4,5}$, Jae-Gook Shin, MD, \\ $\mathrm{PhD}^{4,5}$ and Kiyuk Chang, MD, PhD 3
}

\begin{abstract}
Purpose: We evaluated the incremental prognostic value of combining the CYP2C19 poor metabolizer (PM) and ABCB1 3435 TT for adverse clinical outcomes over conventional risk factors in a percutaneous coronary intervention (PCI) cohort.

Methods: We enrolled 2,188 patients. The primary end point was a composite of death from any cause, nonfatal myocardial infarction (MI), and stroke during 1-year follow-up. The population was stratified into the following four groups: CYP2C19 EM/IM+ABCB1 3435 CC/CT, CYP2C19 EM/IM+ABCB1 3435 TT, CYP2C19 PM+ABCB1 $3435 \mathrm{CC} / \mathrm{CT}$, and CYP2C19 PM+ABCB1 $3435 \mathrm{TT}$.
\end{abstract}

Results: A total of 87 (3.97\%) primary end-point events occurred (64 deaths, 8 non-fatal MIs and 15 strokes). Multivariate Cox analysis indicated that $C Y P 2 C 19 \mathrm{PM}+A B C B 13435 \mathrm{TT}$ status was a significant predictor of the primary end point (hazard ratio $=4.51,95 \%$ confidence interval $(\mathrm{CI})=1.92-10.58)$. However, addition of com- bined genetic status to the clinical risk model did not improve the model discrimination $(\mathrm{C}$-statistic $=0.786(95 \% \mathrm{CI}=0.734-0.837)$ to 0.785 (95\% CI $=0.733-0.838)$ ) or risk reclassification (categorical net reclassification improvement $(0.040, P=0.32)$, integrated discrimination improvement $(0.021, P=0.026))$.

Conclusions: In a real-world East Asian PCI population taking clopidogrel, although the concurrent presence of CYP2C19 PM and $A B C B 1 \mathrm{TT}$ is a strong independent predictor of adverse outcomes, the combined status of two at-risk variants does not have an incremental prognostic value beyond that of the conventional clinical risk factors.

Genet Med advance online publication 23 December 2015

Key Words: ABCB1 3435 TT polymorphism; clopidogrel; CYP2C19 poor metabolizer; genotyping; reclassification

\section{INTRODUCTION}

The identification of individuals at risk of future adverse cardiovascular outcomes is important after percutaneous coronary intervention (PCI). Thus, the use of novel risk markers such as genetic polymorphisms to augment standard risk algorithms, including conventional clinical risk factors, has attracted increasing attention in recent years.

Dual antiplatelet therapy with aspirin and clopidogrel is the current standard of treatment after PCI with drug-eluting stent to reduce the rate of recurrent ischemic events. ${ }^{1,2}$ However, considerable interindividual variability exists in response to clopidogrel, and those with high on-clopidogrel platelet reactivity are at increased risk of cardiovascular events. ${ }^{3,4}$ Several clinical, genetic, and cellular factors have been suggested as mechanisms for clopidogrel response variability. ${ }^{5}$ One of the common causes of individual variations in drug response is genetic polymorphism associated with drug absorption and metabolism. ${ }^{6-8}$

Clopidogrel is a prodrug that requires active enteric absorption and conversion into an active metabolite in the liver. ${ }^{9}$ A key protein involved in clopidogrel absorption is the intestinal efflux transporter P-glycoprotein which is encoded by the $A B C B 1$ gene. ${ }^{8}$ The genetic variant $3435 \mathrm{C}>\mathrm{T}$ is associated with impaired function of the intestinal drug-efflux transporter. Previous findings suggest that individuals with the $A B C B 13435$ TT genotype have reduced concentrations of the active drug metabolite ${ }^{8}$ and increased rates of adverse clinical 


\section{ORIGINAL RESEARCH ARTICLE}

outcomes compared with CC and CT carriers. ${ }^{10,11}$ After intestinal absorption, biotransformation of clopidogrel into active thiol metabolite is accomplished by hepatic cytochrome P450 isoenzymes such as CYP2C19. According to previous studies, the CYP2C19 loss-of-function (LOF) alleles ( ${ }^{\star} 2$ and ${ }^{\star} 3$ alleles) result in a decreased serum concentration of active metabolite, which causes high on-clopidogrel platelet reactivity and then translates into a higher rate of subsequent cardiovascular events than noncarriers. ${ }^{10-14}$ Of note, individuals carrying two LOF alleles, who are considered CYP2C19 poor metabolizers (PMs), showed the worst pharmacokinetic/pharmacodynamic profiles for clopidogrel and the highest risk of cardiovascular outcomes compared with those with one LOF allele or none. ${ }^{10,13,14}$ Because the metabolizing enzyme and drug transporter function together instead of separately in pharmacokinetics, it may be reasonable to assume that the evaluation of both the CYP2C19 PM status and $A B C B 13435$ TT might improve the ability to predict adverse clinical outcomes in patients taking clopidogrel after PCI. However, there is a paucity of data on the combined effect of these two at-risk variants.

Meanwhile, although there is evidence that CYP2C19 PM and $A B C B 13435$ TT are associated with adverse outcomes, the current guideline does not recommend routine genetic testing to assess clopidogrel metabolism for patients considering treatment because of a lack of evidence that the association is clinically important. ${ }^{15}$ To demonstrate clinical usefulness, a novel risk marker should provide clinically significant prognostic value above and beyond that provided by conventional risk factors. The traditional metrics for evaluating the incremental values of novel markers for risk prediction involve a comparison of the C-statistic before and after the addition of the new markers to a baseline risk model. ${ }^{16}$ However, the C-statistic is relatively insensitive to change and may not increase appreciably even when a new marker is statistically significant and independently associated with risk. ${ }^{17}$ Therefore, alternative novel statistical metrics have recently been proposed to assess whether the addition of a new risk marker results in a substantial proportion of individuals being moved ("reclassified") across a predefined risk threshold. These metrics include net reclassification improvement (NRI) and integrated discrimination improvement (IDI), which quantify the appropriateness and amount of overall reclassification..$^{18}$ These two indexes are more powerful than the $\mathrm{C}$-statistic for comparing two prediction models. ${ }^{18}$

To the best of our knowledge, no data are available regarding the additional prognostic value of the combination of these at-risk variants over traditional clinical risk factors in a realworld East Asian PCI population or in other ethnic groups. Therefore, the aim of our study was to evaluate the incremental prognostic value of the combination of the CYP2C19 PM and $A B C B 13435$ TT for adverse clinical outcomes over conventional clinical risk factors using traditional (C-statistic) and novel (NRI, IDI) statistical metrics in an East Asian PCI cohort taking clopidogrel.

\section{MATERIALS AND METHODS \\ Study population}

We enrolled 2,188 patients undergoing drug-eluting stent implantation at two affiliated hospitals of the Catholic University of Korea from January 2005 to December 2009. For this study, patients were considered eligible if they were older than 18 years of age, were maintained on dual antiplatelet therapy with aspirin and clopidogrel after stenting, and provided informed consent for genotyping. Patients were included regardless of the clinical presentation (stable angina or acute coronary syndrome) at the time of the procedure. The exclusion criteria were as follows: contraindications to aspirin or clopidogrel treatment $(n=20)$, known platelet-function disorder or thrombocytopenia (platelet count $\left.<80 \times 10^{3} / \mu \mathrm{l}\right)(n=4)$, or malignancy $(n=2)$. At least 5 days before elective PCI, $100 \mathrm{mg} /$ day aspirin was prescribed, and $250-325 \mathrm{mg}$ aspirin was prescribed as a loading dose before primary PCI. All patients received a loading dose of 300-600 mg clopidogrel. Postprocedural therapy consisted of $100 \mathrm{mg}$ aspirin once daily and $75 \mathrm{mg}$ clopidogrel once daily. The study protocol was approved by the institutional review boards at each participating hospital, and all patients provided written informed consent. The compliance of medication and the presence of adverse clinical events were assessed at every outpatient clinic visit. Most of the data were collected with the use of electronic charts. Complete follow-up data for major clinical events were obtained in $98.1 \%$ of patients. All outcomes of interest were confirmed by source document and were centrally adjudicated by a local events committee at the Cardiovascular Center of Seoul St. Mary's Hospital, Seoul, whose members were unaware of patient status. For validation of the complete follow-up data, censored survival data (for death or survival) were obtained through 30 September 2010 from the database of the National Health Insurance Corporation in Korea using unique personal identification numbers. This study is registered with ClinicalTrials.gov (NCTO1239914).

\section{Genotyping}

The presence of CYP2C19*2 (rs4244285), CYP2C19*3 (rs4986893), and $A B C B 13435 \mathrm{C}>\mathrm{T}$ (rs1045642) was determined via the single-base extension method using an $\mathrm{ABI}$ PRISM genetic analyzer (Applied Biosystems, Foster City, CA). Hardy-Weinberg disequilibrium was not found to be significant for any of the tested genotypes $(P>0.05)$. For the CYP2C19 polymorphism, the patients were classified into three groups according to their predicted metabolizer phenotypes, as determined by the number of LOF alleles: extensive metabolizer $\left(\mathrm{EM} ;{ }^{*} 1{ }^{*} 1\right.$ or $\left.{ }^{\star} /{ }^{*} 17\right)$, intermediate metabolizer (IM; ${ }^{*} 1{ }^{*} 2$ or ${ }^{\star} 1 /{ }^{*} 3$ ), and poor metabolizer $\left(\mathrm{PM} ;{ }^{\star} 2 /{ }^{\star} 2,{ }^{\star} 2 /{ }^{\star} 3\right.$ or $\left.{ }^{\star} 3 /{ }^{*} 3\right)$. For the $A B C B 13435 \mathrm{C}>\mathrm{T}$ polymorphism, the patients were stratified according to the number of $\mathrm{T}$ alleles as follows: CC, CT, and TT. The overall population was categorized into the following four groups according to the presence of the at-risk variants (CYP2C19 PM and $A B C B 1$ TT) of each gene to assess the combined effect of these variants: CYP2C19 EM/IM+ABCB1 
3435 CC/CT, CYP2C19 EM/IM+ABCB1 3435 TT, CYP2C19 $\mathrm{PM}+A B C B 13435 \mathrm{CC} / \mathrm{CT}$, and CYP2C19 $\mathrm{PM}+A B C B 13435 \mathrm{TT}$. The results of genotyping were given to the attending physicians at each hospital for personalization of antiplatelet therapy.

\section{Platelet-function test}

To evaluate the effect of clopidogrel on platelet aggregation, we measured P2Y12 platelet reaction units using the VerifyNow P2Y12 test. To reflect the patients' full responses to clopidogrel and to minimize the effects of acute anticoagulant therapy during PCI, the test was performed on the day of discharge for all patients. Because the test has only been available since June 2008, a limited number of patients $(n=693)$ underwent the platelet-function test during the study period.

\section{Study end points and definitions}

The primary end point was a composite of major adverse cardiac and cerebrovascular events (MACCEs), including death from any cause, nonfatal myocardial infarction (MI), (nonprocedural), and stroke during 1 year of follow-up. According to the current guideline, ${ }^{19}$ dual antiplatelet therapy should be given at least 12 months after PCI. Thereafter, aspirin should be continued indefinitely and clopidogrel can be stopped at the physician's discretion according to the patient's condition. Because genetic polymorphism of CYP2C19 and ABCB1 3435 $\mathrm{C}>\mathrm{T}$ are associated with pharmacokinetics/pharmacodynamics of clopidogrel, all outcomes of interest should be evaluated only during clopidogrel exposure. Thus, we chose the composite of 1 -year adverse outcomes as the primary end point.

\section{Statistical analysis}

Variables are presented as the mean \pm standard deviation (SD) or frequencies (percentage). Continuous variables were compared using Student's $t$-test or one-way ANOVA, and categorical variables were compared using the chi-squared test or Fisher's exact test. To examine the association between the risk factors and the primary end point, we performed univariate and multivariate Cox regression analyses. The cumulative event rates for the primary end point were estimated by the KaplanMeier method according to the specified four combined genetic groups and compared by log-rank tests.

To assess the incremental prognostic value of the addition of genetic risk factors to the conventional clinical risk factors, we created the following two multivariate models: (i) a baseline clinical model including established conventional factors (age, male gender, $\mathrm{MI}$ as an index of diagnosis, chronic kidney disease, prior stroke, and left ventricular ejection fraction $<40 \%$ ) and (ii) a clinical-genetic model containing the covariates of the clinical model plus the four combined genetic groups. The selection of variables for the baseline clinical model was determined by a backward-stepwise technique, evaluating all potential univariate variables $(P<0.20)$ to create a multivariable model containing the variables with a $P<0.05$. We performed several sensitivity analyses that confirmed the consistency of the models (e.g., modeling using the forward selection of variables, interaction analyses, and the rejection of colinearity). The discriminatory abilities of the models were assessed using the C-statistic, and differences in the C-statistic between the two models were compared according to the method of DeLong et al. ${ }^{20}$ The calibration of the two models was tested using the Hosmer-Lemeshow goodness-of-fit test, and model fitness was assessed using the Akaike information criterion. Furthermore, to evaluate whether the addition of the genetic risk factors to the baseline clinical model led to any significant risk reclassification of the primary end point, NRI and IDI were calculated according to the method described by Pencina et al. ${ }^{18}$ To evaluate clinical usefulness, categorical NRIs were applied with prespecified risk categories of $<2.20 \%, 2.20-16.44 \%$, and $\geq 16.44 \%$, which were selected in accordance with the observed primary end-point rate of the present study (3.97\%). Because net reclassification depends on the chosen risk threshold, ${ }^{21}$ the thresholds in the sensitivity analysis were defined as 2.20 and $16.44 \%$, which were more evenly distributed around the event rate of $3.97 \%$. For the combined genetic status, the sample size of our study $(2,188)$ provides $83.4 \%$ power to detect an effect size (Cramer's V) of 0.0735 with $\alpha=0.05$. A $P$ value $<0.05$ was considered statistically significant. Statistical analysis was performed using SPSS 18.0 (SPSS, Chicago, IL).

\section{RESULTS}

\section{Patient characteristics}

A total of 2,188 patients were enrolled in our study. Approximately $60 \%$ of the population had at least one CYP2C19 LOF allele (IM or PM) and 14\% had two LOF alleles (PM). In terms of the prevalence of $A B C B 13435 \mathrm{C}>\mathrm{T}$ polymorphism, $57 \%$ of the population had one or more T-allele (CT or TT) and $13.7 \%$ had two T-alleles (TT). Table 1 shows the baseline characteristics of the overall population according to the presence of MACCEs. The patients with MACCEs were older and had a lower body mass index. They also demonstrated a higher prevalence of hypertension, $\mathrm{MI}$ as an index diagnosis, chronic kidney disease, left main coronary artery disease, the $A B C B 1$ 3435 CT genotype, and the presence of both CYP2C19 PM and $A B C B 13435 \mathrm{TT}$, as well as a greater incidence of prior coronary artery bypass grafting, prior stroke, and a left ventricular ejection fraction of $<40 \%$ compared with those who did not experience MACCEs.

On-clopidogrel platelet reactivity measured by the VerifyNow P2Y12 assay showed a statistically significant gene dose-response effect according to the CYP2C19 polymorphism metabolic status (platelet reaction unit value (mean \pm SD): $200.7 \pm 88.47$ vs. $231.49 \pm 79.87$ vs. $259.67 \pm 85.23$ for EM vs. IM vs. PM, respectively, $P<0.0001$ among the groups) (Supplementary Figure S1a online). However, a gene dose-response effect was not observed for the $A B C B 13435 \mathrm{C}>\mathrm{T}$ polymorphism among the groups (platelet reaction unit value (mean \pm SD): $223.45 \pm 85.27$ vs. $221.36 \pm 84.6$ vs. $232.39 \pm 87.71$ for CC vs. CT vs. TT, respectively, $P=0.56$ among the groups) (Supplementary Figure S1b online). Consequently, when the four combined genetic subsets were assessed, a significant stepwise increase of platelet 
Table 1 Baseline characteristics of the study population according to the presence of the primary end point

\begin{tabular}{|c|c|c|c|c|}
\hline $\begin{array}{l}\text { Clinical characteristics } \\
\text { ( } n=\text { number of patients) }\end{array}$ & $\begin{array}{c}\text { Total } \\
(n=2,188)\end{array}$ & $\begin{array}{l}\text { MACCE }(+) \\
(n=87)\end{array}$ & $\begin{array}{l}\text { MACCE (-) } \\
(n=2,101)\end{array}$ & $P$ \\
\hline Age (years) & $63.5 \pm 10.6$ & $70.2 \pm 10.9$ & $63.2 \pm 10.5$ & $<0.001$ \\
\hline Male & $739(33.7)$ & $23(26.4)$ & $716(34.1)$ & 0.14 \\
\hline $\mathrm{BMI}\left(\mathrm{kg} / \mathrm{m}^{2}\right)$ & $24.7 \pm 3.1$ & $23.1 \pm 2.9$ & $24.7 \pm 3.1$ & $<0.001$ \\
\hline DM & $825(37.7)$ & $39(44.8)$ & $786(37.4)$ & 0.16 \\
\hline HBP & $1,300(59.4)$ & $61(70.1)$ & $1,239(59)$ & 0.038 \\
\hline Current smoking status & $389(17.8)$ & $14(16.1)$ & $375(17.8)$ & 0.67 \\
\hline Family history of $C A D$ & $122(5.6)$ & $3(3.4)$ & $119(5.7)$ & 0.37 \\
\hline Dyslipidemia & $440(20.1)$ & $11(12.6)$ & $429(20.4)$ & 0.12 \\
\hline Prior Ml & $155(7.1)$ & $9(10.3)$ & $146(6.9)$ & 0.23 \\
\hline Prior PCl & $209(9.5)$ & $9(10.3)$ & $200(9.5)$ & 0.78 \\
\hline Prior CABG & $20(0.9)$ & $3(3.4)$ & $17(0.8)$ & 0.01 \\
\hline Prior stroke & $143(6.5)$ & $12(13.8)$ & $131(6.2)$ & 0.005 \\
\hline MI as an index diagnosis & $532(24.3)$ & $42(48.3)$ & $490(23.3)$ & $<0.001$ \\
\hline CKD $\left(e G F R<60 \mathrm{ml} / \mathrm{min} / 1.73 \mathrm{~m}^{2}\right)$ & $96(4.4)$ & $24(27.6)$ & $72(3.4)$ & $<0.001$ \\
\hline LVEF $<40 \%$ & $160(7.3)$ & $24(27.5)$ & $136(6.5)$ & $<0.001$ \\
\hline \multicolumn{5}{|l|}{ Involved vessel } \\
\hline LM & $108(4.9)$ & $10(11.5)$ & $98(4.7)$ & 0.005 \\
\hline LAD & $1,703(77.8)$ & $65(74.7)$ & $1,638(77.9)$ & 0.9 \\
\hline LCX & $1,123(51.3)$ & $50(57.4)$ & $1,073(51)$ & 0.83 \\
\hline RCA & $1,146(52.3)$ & $54(62)$ & $1,092(51.9)$ & 0.12 \\
\hline Stent diameter (mm) & $3.18 \pm 0.5$ & $3.08 \pm 0.8$ & $3.18 \pm 0.47$ & 0.24 \\
\hline Stent length (mm) & $27.8 \pm 13.7$ & $27.9 \pm 14.4$ & $27.8 \pm 13.7$ & 0.94 \\
\hline ACC/AHA type B2/C lesion & $1,274(58.2)$ & $55(63.2)$ & $1,219(58)$ & 0.76 \\
\hline \multicolumn{5}{|l|}{ Discharge medication } \\
\hline Aspirin & $2,160(98.7)$ & $86(98.8)$ & $2,074(98.1)$ & 0.71 \\
\hline Statin & $1,933(88.3)$ & $56(86.2)$ & $1,877(89.4)$ & 0.4 \\
\hline$\beta$-blocker & $1,506(68.9)$ & $59(67.8)$ & $1,447(68.9)$ & 0.83 \\
\hline $\mathrm{CCB}$ & $414(18.9)$ & $20(22.9)$ & $394(18.8)$ & 0.38 \\
\hline ACEi/ARB & $1,787(81.7)$ & $65(74.7)$ & $1,722(82)$ & 0.17 \\
\hline PPI & $454(20.7)$ & $23(26.4)$ & $431(20.5)$ & 0.45 \\
\hline CYP2C19 metabolic phenotype & & & & 0.067 \\
\hline $\operatorname{EM}(* 1 / * 1, * / * 17)$ & $872(39.8)$ & $27(31.0)$ & $845(40.2)$ & \\
\hline $\operatorname{IM}(* 1 / * 2, * 1 / * 3)$ & $1,003(45.8)$ & $41(47.1)$ & $962(45.8)$ & \\
\hline $\mathrm{PM}(* 2 / * 2, * 2 / * 3, * 3 / * 3)$ & $313(14.3)$ & $19(21.8)$ & $294(14.0)$ & \\
\hline$A B C B 13435 C>T$ & & & & 0.019 \\
\hline CC & $925(42.2)$ & $44(50.6)$ & $881(41.9)$ & 0.11 \\
\hline $\mathrm{CT}$ & $946(43.2)$ & $26(29.9)$ & $938(44.6)$ & 0.007 \\
\hline$\pi$ & $299(13.7)$ & $17(19.5)$ & $282(13.4)$ & 0.104 \\
\hline Combined genetic status $^{\mathrm{a}}$ & & & & 0.008 \\
\hline CYP2C19 EM/IM+ABCB1 3435 CC/CT & $1,621(74.1)$ & $57(65.5)$ & $1,564(74.4)$ & 0.063 \\
\hline CYP2C19 EM/IM+ABCB1 3435 TT & $254(11.6)$ & $11(12.6)$ & $243(12)$ & 0.434 \\
\hline CYP2C19 PM+ABCB1 3435 CC/CT & $268(12.2)$ & $13(14.9)$ & $255(12.6)$ & 0.758 \\
\hline CYP2C19 PM+ABCB1 $3435 \mathrm{TT}$ & $45(2.0)$ & $6(6.8)$ & $39(1.9)$ & 0.001 \\
\hline
\end{tabular}

The values are expressed as the mean \pm SD or $n(\%)$ of patients.

${ }^{a}$ According to the presence of CYP2C19 PM status and ABCB1 3435 TT genotype.

ACC/AHA, American College of Cardiology/American Heart Association classification; ACEi/ARB, angiotensin converting enzyme inhibitor/angiotensin receptor blocker; BMI, body mass index; $C A B G$, coronary artery bypass grafting; $C A D$, coronary artery disease; $C C, A B C B 13435 C>T$ wild allele; $C C B$, calcium channel blocker; $C K D$, chronic kidney disease; CT, ABCB1 3435C>T heterozygotic allele; CYP, cytochrome P450; DM, diabetes mellitus; eGFR, estimated glomerular filtration rate; EM, extensive metabolizer; HBP, hypertension; IM, intermediate metabolizer; LAD, left anterior descending artery; LCX, left circumflex artery; LM, left main coronary artery; LVEF, left ventricular ejection fraction; MI, myocardial infarction; $\mathrm{PCl}$, percutaneous coronary intervention; PM, poor metabolizer; PPI, proton pump inhibitor; RCA, right coronary artery; $T \mathrm{~T}, A B C B 13435 C>\mathrm{T}$ homozygotic allele. 
reactivity was observed (platelet reaction unit value (mean \pm SD): $217.43 \pm 84.64$ vs. $217.37 \pm 90.08$ vs. $253.24 \pm 79.96$ vs. $287.74 \pm 49.01$ for $C Y P 2 C 19 \mathrm{EM} / \mathrm{IM}+A B C B 13435 \mathrm{CC} / \mathrm{CT}$ vs. $C Y P 2 C 19 \mathrm{EM} / \mathrm{IM}+A B C B 13435 \mathrm{TT}$ vs. $C Y P 2 C 19 \mathrm{PM}+A B C B 1$ $3435 \mathrm{CC} / \mathrm{CT}$ vs. CYP2C19 PM+ABCB1 3435 TT, respectively, $P<0.001$ among the groups) (Supplementary Figure S1c online).

\section{Association of genetic and clinical risk factors with MACCE} Table 2 shows the incidences of the primary end-point events according to genetic analysis. During the 1-year follow-up, a total of 87 (3.97\%) primary end-point events occurred, including 64 deaths, 8 non-fatal MIs, and 15 strokes. Among the four combined genetic groups, the patients carrying both CYP2C19 PM and $A B C B 13435$ TT showed the highest incidence of allcause death, stroke, and composite of MACCEs compared with those in the other groups $(P<0.01)$. Detailed causes of death according to the genetic variants is presented in Supplementary Table S1 online.

The observed hazard ratios (HRs) for the risk factors associated with the MACCEs are summarized in Table 3. Univariate Cox analysis revealed that age, body mass index, hypertension, MI as an index diagnosis, chronic kidney disease, prior stroke, a left ventricular ejection fraction of $<40 \%$, left main coronary artery disease, CYP2C19 PM, and the concurrent presence of both CYP2C19 PM and ABCB1 3435 TT were significant univariable predictors of 1 -year MACCEs. It is noteworthy that although the $A B C B 13435 \mathrm{TT}$ was not a significant univariable predictor, when we considered combined status of $C Y P 2 C 19$ PM and $A B C B 13435$ TT, the HR of patients who had both atrisk variants increased remarkably compared to that of each atrisk variant. Multivariate analysis showed that age, male gender, $\mathrm{MI}$ as an index diagnosis, chronic kidney disease, prior stroke, a left ventricular ejection fraction of $<40 \%$, and also the concurrent presence of both CYP2C19 PM and ABCB1 3435 TT were independent prognostic factors for MACCEs. Patients with both CYP2C19 PM and ABCB1 3435 TT had an approximately 4.5-fold higher HR for MACCEs ( $\mathrm{HR}=4.51,95 \%$ confidence interval $(\mathrm{CI})=1.92-10.58)$ compared with those who did not carry either at-risk variant. However, there was no significant interaction between CYP2C19 PM and ABCB1 3435 TT ( $P$ for interaction $=0.052$ ).

Figure 1 shows the 1-year cumulative event rates of MACCEs for the four combined genetic groups. Among the groups, a significant stepwise increased risk of MACCEs was observed according to the presence of the CYP2C19 PM and ABCB1 $3435 \mathrm{TT}$, with the highest event rate observed for the group carrying both at-risk variants, as follows: $13.8 \%$ (crude $\mathrm{HR}=$ $3.98,95 \% \mathrm{CI}=1.71-9.21$ ), $4.9 \%$ (crude $\mathrm{HR}=1.38,95 \% \mathrm{CI}=$ $0.75-2.52$ ), $4.4 \%$ (crude $\mathrm{HR}=1.22,95 \% \mathrm{CI}=0.64-2.33$ ), and $3.6 \%$ (reference, $\log \operatorname{rank} P=0.007$ ) for the individuals with both CYP2C19 PM and ABCB1 3435 TT, those with CYP2C19 PM but lacking $A B C B 13435$ TT, those with $A B C B 13435$ TT but lacking CYP2C19 PM, and those lacking both variants, respectively.

\section{Incremental prognostic value of genetic risk factors over clinical risk factors}

Table 4 shows the results of analysis using calibration, discrimination, and reclassification metrics to evaluate the incremental usefulness of genetic risk factors over clinical risk factors for the prediction of MACCEs.

\section{Calibration and model fit}

The $P$ values for the Hosmer-Lemeshow statistics were 0.79 for the baseline clinical model and 0.89 for the clinical-genetic model, signifying that both models were well calibrated. The Akaike information criterion was lower in the model containing combined genetic factors than in the model including the clinical factors alone.

\section{Discrimination}

Both the baseline clinical model (C-statistic $=0.786,95 \%$ $\mathrm{CI}=0.734-0.837, P=0.03)$ and the clinical-genetic model $(\mathrm{C}$-statistic $=0.785,95 \% \mathrm{CI}=0.733-0.838, P=0.03)$ provided significant discriminatory information for the prediction of the primary end-point events. However, although the individuals with $C Y P 2 C 19$ PM and $A B C B 13435$ TT had a 4.5-fold

Table 2 Incidence of primary end point according to genetic analysis

\begin{tabular}{|c|c|c|c|c|c|c|c|c|}
\hline & \multicolumn{2}{|c|}{$\begin{array}{l}\text { CYP2C19 metabolic } \\
\text { phenotype }\end{array}$} & \multicolumn{2}{|c|}{$\begin{array}{c}A B C B 13435 C>T \\
\text { genotype }\end{array}$} & \multicolumn{4}{|c|}{$\begin{array}{c}\text { Combined } \\
\text { genetic status }\end{array}$} \\
\hline Any death & $51(2.7)$ & $13(4.2)$ & $54(2.9)$ & $10(3.3)$ & $45(2.7)$ & $6(2.4)$ & $8(2.9)$ & $5(7.7)^{d}$ \\
\hline Nonfatal MI & $7(0.4)$ & $1(0.3)$ & $6(0.3)$ & $2(0.7)$ & $6(0.3)$ & $1(0.4)$ & $1(0.4)$ & 0 \\
\hline Stroke & $10(0.5)$ & $5(1.6)^{c}$ & $10(0.5)$ & $5(1.7)^{c}$ & $6(0.4)$ & $4(1.6))$ & $4(1.5)$ & $1(2.2)^{c}$ \\
\hline $\begin{array}{l}\text { Stent thrombosis } \\
\text { (definite or probable) }\end{array}$ & $15(0.8)$ & $3(1)$ & $15(0.8)$ & $3(1)$ & $12(0.7)$ & $3(1.2)$ & $3(1.1)$ & 0 \\
\hline Bleeding (major or minor) & $14(0.7)$ & $2(0.6)$ & $13(0.7)$ & $3(1)$ & $12(0.7)$ & $2(0.8)$ & $1(0.4)$ & $1(2.2)$ \\
\hline
\end{tabular}

${ }^{a}$ According to the Academic Research Consortium criteria. ${ }^{b}$ According to the thrombolysis in myocardial infarction criteria. ${ }^{c} P<0.05 ;{ }^{d} P<0.01$.

EM, extensive metabolizer; IM, intermediate metabolizer; PM, poor metabolizer. 
Table 3 Predictors of primary end point by univariate and multivariate Cox regression analyses

\begin{tabular}{|c|c|c|}
\hline & Univariate analysis & $\begin{array}{l}\text { Multivariate } \\
\text { analysis }\end{array}$ \\
\hline Covariate & HR $(95 \% \mathrm{Cl})$ & HR $(95 \% \mathrm{Cl})$ \\
\hline Age & $1.07(1.05-1.10)^{\ddagger}$ & $1.06(1.04-1.09)^{\ddagger}$ \\
\hline Male & $1.43(0.89-2.30)$ & $1.92(1.17-3.16) *$ \\
\hline BMI & $0.85(0.81-0.90)^{\ddagger}$ & $0.93(0.86-1.01)$ \\
\hline $\mathrm{HBP}$ & $1.61(1.02-2.55)^{\star}$ & $1.15(0.67-1.97)$ \\
\hline DM & $1.36(0.89-2.07)$ & $1.06(0.65-1.72)$ \\
\hline Dyslipidemia & $0.57(0.30-1.07)$ & $0.83(0.39-1.72)$ \\
\hline Current smoking status & $0.89(0.50-1.56)$ & $0.84(0.44-1.60)$ \\
\hline Ml as an index diagnosis & $3.01(1.97-4.58)^{\ddagger}$ & $2.10(1.34-3.29)^{\ddagger}$ \\
\hline CKD & $9.88(6.17-15.82)^{+}$ & $5.62(3.42-9.23)^{\ddagger}$ \\
\hline Prior Ml & $1.54(0.77-3.07)$ & $1.32(0.56-2.44)$ \\
\hline Prior stroke & $2.35(1.28-4.33)^{\dagger}$ & $2.05(1.11-3.78)^{*}$ \\
\hline LVEF $<40 \%$ & $4.95(3.03-8.09)^{\ddagger}$ & $2.29(1.35-3.87)^{\dagger}$ \\
\hline LMD & $2.56(1.32-4.97)^{\dagger}$ & $1.63(0.81-3.32)$ \\
\hline $\begin{array}{l}\text { ACC/AHA type B2+C } \\
\text { lesion }\end{array}$ & $1.21(0.78-1.86)$ & $1.18(0.53-1.24)$ \\
\hline Stent diameter (mm) & $0.70(0.48-1.02)$ & $0.54(0.32-1.01)$ \\
\hline Stent length (mm) & $1.00(0.99-1.02)$ & $1.21(0.87-1.12)$ \\
\hline \multicolumn{3}{|c|}{ CYP2C19 metabolic phenotype } \\
\hline EM & 1 & \\
\hline $\mathrm{IM}$ & $1.26(0.78-2.06)$ & \\
\hline PM & $1.94(1.07-3.48)^{*}$ & \\
\hline PM (vs. EM/IM) & $1.69(1.02-2.81)^{\star}$ & \\
\hline \multicolumn{3}{|l|}{$A B C B 13435 C>T$ genotype } \\
\hline CC & 1 & \\
\hline $\mathrm{CT}$ & $0.55(0.34-0.91)$ & \\
\hline TT & $1.20(0.68-2.14)$ & \\
\hline $\mathrm{TT}$ (vs. CC/CT) & $1.53(0.90-2.61)$ & \\
\hline \multicolumn{3}{|c|}{ Combined status based on CYP2C19 PM and ABCB1 343 TT } \\
\hline $\begin{array}{l}\text { CYP2C19 EM/IM + } \\
\text { ABCB1 } 3435 \text { CC/CT }\end{array}$ & 1 & 1 \\
\hline $\begin{array}{l}\text { CYP2C19 EM/IM + } \\
\text { ABCB1 } 3435 \mathrm{TT}\end{array}$ & $1.22(0.64-2.33)$ & $1.23(0.86-2.87)$ \\
\hline $\begin{array}{l}\text { CYP2C19PM + ABCB1 } \\
3435 \text { CC/CT }\end{array}$ & $1.38(0.75-2.52)$ & $1.57(0.64-2.34)$ \\
\hline $\begin{array}{l}\text { CYP2C19PM + ABCB1 } \\
3435 \mathrm{TT}\end{array}$ & $3.98(1.71-9.21)^{\ddagger}$ & $4.51(1.92-10.58)^{\ddagger}$ \\
\hline
\end{tabular}

${ }^{*} P<0.05 ;{ }^{+} P<0.01 ;{ }^{\ddagger} P<0.001$.

ACC/AHA, American College of Cardiology/American Heart Association classification; $A C E$ I/ARB, angiotensin converting enzyme inhibitor/angiotensin receptor blocker; BMI, body mass index; $C A B G$, coronary artery bypass grafting; $C A D$, coronary artery disease; $C C B$, calcium channel blocker; $C K D$, chronic kidney disease; CYP, cytochrome P450; DM, diabetes mellitus; eGFR, estimated glomerular filtration rate; $\mathrm{EM}$, extensive metabolizer; HBP, hypertension; IM, intermediate metabolizer; LAD, left anterior descending artery; LCX, left circumflex artery; LM, left main coronary artery; LVEF, left ventricular ejection fraction; MI, myocardial infarction; $\mathrm{PCl}$, percutaneous coronary intervention; PM, poor metabolizer; PPI, proton pump inhibitor; RCA, right coronary artery.

higher relative risk than those lacking both variants according to multivariate Cox analysis, the addition of combined genetic risk factors to the baseline clinical model did not increase the C-statistic (estimated difference in the C-statistic $=-0.001 ; 95 \%$ $\mathrm{CI}=-0.073-0.074, P=0.99)$ for predicting the primary end

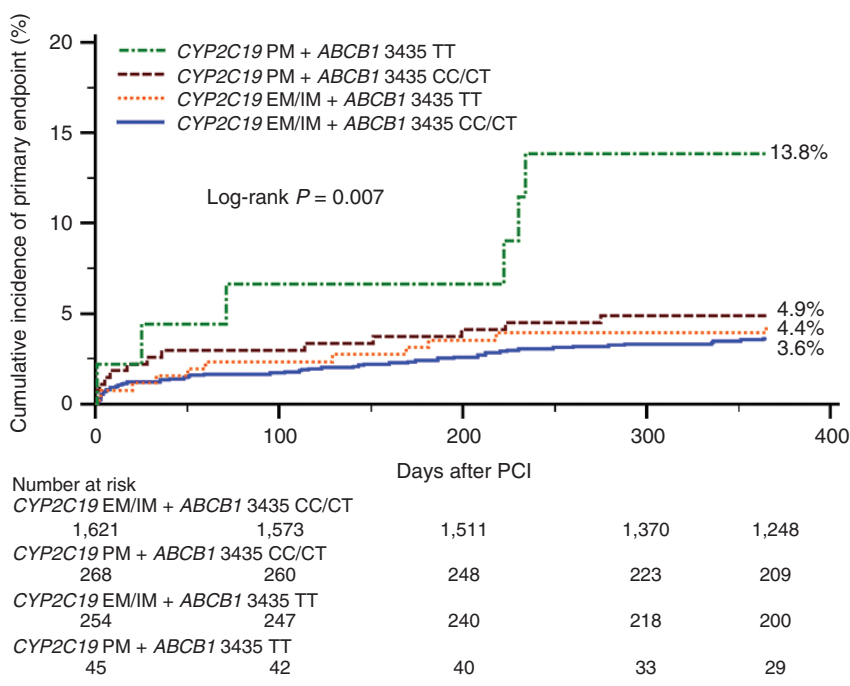

Figure 1 Kaplan-Meier curves of the cumulative event rate of the primary end point according to the combination of CYP2C19 PM status and $A B C B 13435$ TT genotype. Among the four groups, a significant stepwise increased risk of the primary end point was observed according to the presence of CYP2C19 PM status and ABCB1 3435 TT genotype.

point event. Similarly, when CYP2C19 PM and ABCB1 3435 TT were added to the baseline clinical model, the C-statistic did not increase significantly.

\section{Reclassification}

The addition of the combined genetic factors to the baseline model also did not yield any significant further improvements in risk classification for the prediction of 1-year MACCEs. Although the absolute IDI $(0.021,95 \% \mathrm{CI}=0.003-0.040, P=$ $0.02)$ and relative IDI $(18 \%, 95 \% \mathrm{CI}=6.1-30.0 \%, P=0.003)$ were statistically significant, the categorical NRI $(0.040,95 \%$ CI $=-0.039-0.119, P=0.32)$ and continuous NRI $(0.192,95 \% \mathrm{CI}$ $=-0.011-0.395, P=0.07)$ were not. In addition, the majority of risk reclassification occurred in the non-MACCE group. The net upward reclassification for the patients who experienced an adverse event was $0 \%$, and the net downward reclassification for those who did not was 4\% (Supplementary Table S2 online). Although these results were not statistically significant, they demonstrated that adding the combined genetic variables to the baseline clinical model better identified the patients at low risk.

\section{DISCUSSION}

To the best of our knowledge, this is the first study evaluating the incremental prognostic value of the combination of the CYP2C19 PM and ABCB1 3435 TT alleles over conventional risk factors in a routine clinical practice of a PCI cohort on clopidogrel. Our study demonstrated that although the concurrent presence of both CYP2C19 PM and ABCB1 3435 TT was a strong independent predictor of 1-year MACCEs, adding the combined status of both at-risk variants to a baseline clinical model containing traditional risk factors did not provide significant incremental prognostic value. 


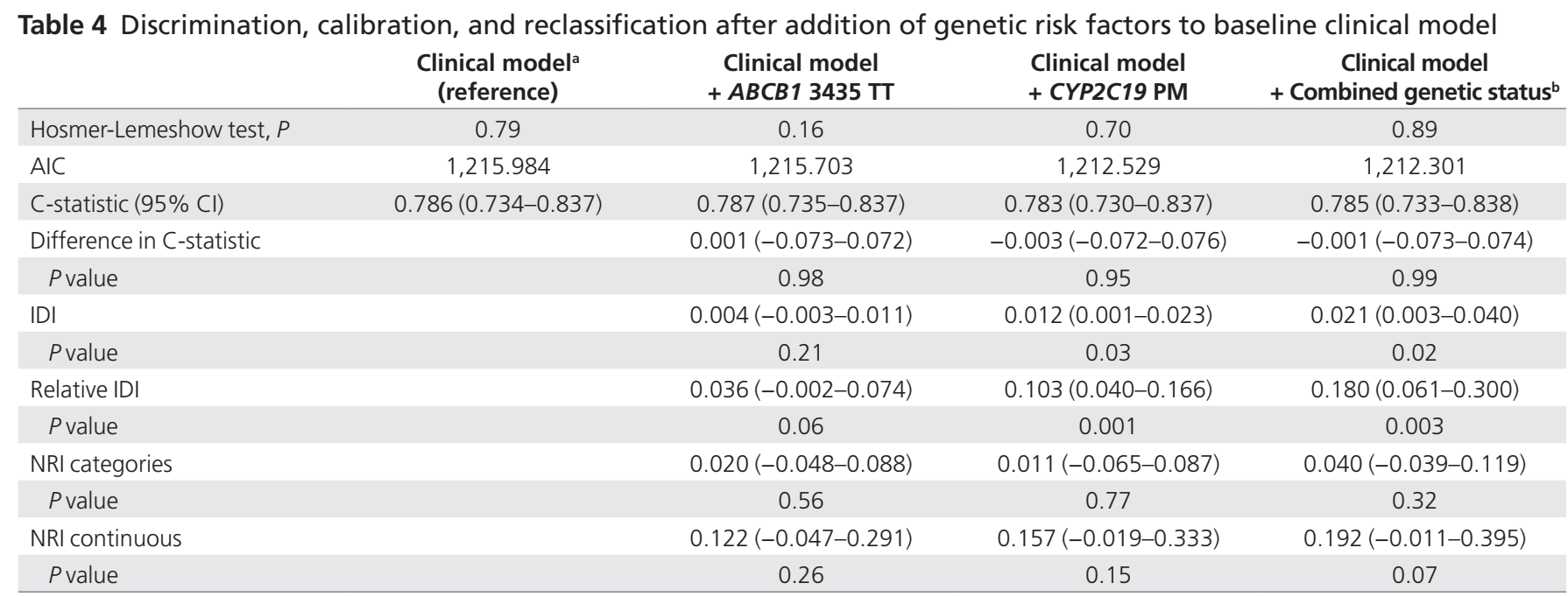

AIC, Akaike information criterion; IDI, integrated discrimination improvement; NRI, net reclassification improvement.

ancluding age, male gender, myocardial infarction as an index diagnosis, chronic kidney disease, previous stroke history, and left ventricular ejection fraction $<40 \%$. lncluding CYP2C19 EM/IM + ABCB1 3435 CC/CT, CYP2C19 EM/IM + ABCB13435 TT, CYP2C19 PM + ABCB1 3435 CC/CT, and CYP2C19 PM + ABCB1 3435 TT.

For the accurate prediction of future cardiovascular events after PCI, several established risk factors, such as age, gender, hypertension, smoking status, DM, and dyslipidemia have been incorporated into a statistical model for risk prediction. However, these factors do not fully explain future cardiovascular events. In a PCI population taking clopidogrel, the interest in genetic testing for clopidogrel metabolism has grown in recent years to augment standard risk algorithms. Based on the prior studies, the CYP2C19 PM status and ABCB1 3435 TT genotype were the main variants of interest for clopidogrel treatment. In the context of the clinical usefulness of genetic testing in PCI patients on clopidogrel, it is reasonable to consider both CYP2C19 PM and ABCB1 3435 TT simultaneously because the metabolizing enzyme and drug transporter function together to elicit a response to clopidogrel. However, there is a paucity of data regarding the combined effect of these two at-risk variants, and no data are available to evaluate the incremental prognostic value of their combined effect over conventional clinical risk factors.

In the present study, CYP2C19 PM status was significantly associated with a 1-year MACCEs, but the $A B C B 13435$ TT was not. According to the prior studies, there is robust data demonstrating the significant association between the CYP2C19 polymorphism and adverse clinical outcomes, whereas the results on the association between $A B C B 13435 \mathrm{C}>\mathrm{T}$ polymorphism and clinical outcomes are mixed..$^{10-12,22}$ In our study, the power to detect significant differences in 1-year MACCEs for the variants of $A B C B 13435 \mathrm{C}>\mathrm{T}$ was limited (35.8\% to detect an effect size 0.0341 with $\alpha=0.05$ ), thus our findings may not be conclusive for this issue. However, although no significant association exists between the $A B C B 13435$ TT and adverse clinical outcomes in our study, when the $A B C B 13435$ TT was combined with the CYP2C19 PM, the on-clopidogrel platelet reactivity and the $\mathrm{HR}$ of patients with both at-risk variants increased remarkably compared to those of each at-risk variant.
Moreover, the individuals carrying both at-risk variants showed the highest event rate compared to those with other combined genetic subsets. This finding is in agreement with the results of two large clinical studies..$^{10,11}$

Our finding demonstrated that patients with both CYP2C19 $\mathrm{PM}$ and $A B C B 13435 \mathrm{TT}$ showed a significantly higher HR (4.51, $P<0.0001)$ for MACCEs than those who did not carry either at-risk variant in multivariable regression analysis. Nevertheless, as expected, the addition of the combined genetic status to the baseline clinical model did not yield further improvements in model discrimination according to the changes in the C-statistic. Furthermore, the new model including the genetic factors did not better reclassify at-risk individuals compared with the baseline clinical model, as determined by assessments of NRI and IDI. The most basic requirement for evaluating the clinical value of a novel risk marker is that the association between the new marker and the outcomes of interest should be statistically significant when tested as a predictor of future adverse events. ${ }^{16}$ Most studies regarding the role of CYP2C19 and ABCB1 $3435 \mathrm{C}>\mathrm{T}$ polymorphism on adverse clinical outcomes in PCI population have reported their results based on the basic association measure such as HRs and odds ratios. However, although strong association may be observed between new markers and clinical outcomes in a multivariate model, it is widely accepted that basic association measures alone are insufficient for determining prognostic value. ${ }^{16}$ Therefore, a more important requirement for a novel marker is that it improves risk prediction over established risk factors, i.e., that novel markers should provide incremental prognostic information. For this, we performed rigorous statistical analysis using traditional C-statistics and novel reclassification metrics such as NRI and IDI. However, our study failed to demonstrate the additional prognostic value of a combined set of two genetic variants over traditional risk factors as a novel risk marker. 
The goal of measuring risk markers is not simply to ascertain an individual's risk; rather, it is to use the risk assessment to guide appropriate therapy and thereby improve clinical outcomes. ${ }^{16}$ Considering this perspective, our findings highlighted the limitations of the current genetic testing of patients in a PCI population taking clopidogrel for predicting adverse clinical outcomes. Our findings also supported the current guidelines, which do not recommend routine genetic testing to assess clopidogrel metabolism for patients considering treatment because of the poor sensitivity and low positive predictive value for identifying "high-risk" patients. ${ }^{15,23}$ In fact, it is very difficult to perform large randomized studies guided by genetic testing. No data are available regarding randomized trials for genotype-guided antiplatelet therapy. Two ongoing randomized studies compare CYP2C19 genotype-guided antiplatelet therapy to a nontailored strategy in PCI patients. ${ }^{24,25}$ In this regard, we believe that the findings of the present observational study could supply additional evidence regarding the clinical appropriateness of the current genetic testing to assess clopidogrel metabolism. In addition, our results also reemphasize that the traditional risk factors are still strong predictors of a broad cardiovascular end point. ${ }^{26}$

\section{Study limitations}

There were several limitations of our analysis. First, this study enrolled only East Asians. Because the effect of a genetic polymorphism on clinical events can differ depending on genetic background or environment, ${ }^{27}$ it may be inappropriate to generalize the results of this study to other ethnic groups. Second, due to the relatively low event rate and low prevalence of carrier with CYP2C19 PM + ABCB1 3435 TT genotype, there is a possibility that the addition of combined genetic status would not alter the prediction results using $\mathrm{C}$-statistics. Therefore, because these factors may make it difficult to draw definite conclusions on the current issues, our findings should be considered with caution until additional large clinical studies with long-term follow-ups replicate our findings. However, with regard to the low event rates of adverse clinical outcomes, numerous clinical data have shown that East Asians may have even lower rates of post-PCI adverse cardiovascular outcomes compared with Westerners. ${ }^{28,29}$ Indeed, the overall 1-year rates of adverse clinical outcomes in other Asian PCI studies are similarly low to those in our study: bleeding complications $(0.2-1.5 \%)$, stent thrombosis $(\sim 0.5 \%)$, and the composite of death, non-fatal MI, and stroke ( 3.0\%). ${ }^{30-33}$ Despite the limitation of the event rates, this study is the largest genetic study to evaluate the incremental predictive value of the combination of the CYP2C19 PM and $A B C B 13435$ TT over traditional risk factors in PCI patients. Therefore, we believe that our results may reflect the situation of routine clinical practice in this population. Third, we performed platelet-function tests on a limited number of patients ( $n=693 ; 31.6 \%$ of the entire study population) because of the late availability of the VerifyNow P2Y12 assay during patient enrollment. Therefore, precisely evaluating the pharmacodynamic aspects of clopidogrel with respect to the polymorphism of the CYP2C19 and the $A B C B 13435 \mathrm{C}>\mathrm{T}$ might be biased in this population. Finally, our study is retrospective and therefore subject to the limitations inherent to such analyses.

\section{Conclusions}

Although the concurrent presence of CYP2C19 PM and $A B C B 1$ TT is a strong independent predictor of 1-year MACCEs, the combined status of these two at-risk variants does not have incremental prognostic value beyond that of conventional clinical risk factors in a real-world East Asian PCI population taking clopidogrel. However, to draw definite conclusions on the current issues, further large-scale multinational collaboration studies are essential.

\section{SUPPLEMENTARY MATERIAL}

Supplementary material is linked to the online version of the paper at http://www.nature.com/gim.

\section{ACKNOWLEDGMENT}

We thank MI Sun Park at the Clinical Research Coordinating Center for statistical consultation.

\section{DISCLOSURE}

The authors declare no conflict of interest.

\section{REFERENCES}

1. Chen ZM, Jiang LX, Chen YP, et al.; COMMIT (ClOpidogrel and Metoprolol in Myocardial Infarction Trial) collaborative group. Addition of clopidogrel to aspirin in 45,852 patients with acute myocardial infarction: randomised placebo-controlled trial. Lancet 2005;366:1607-1621.

2. Mehta SR, Yusuf S, Peters RJ, et al.; Clopidogrel in Unstable angina to prevent Recurrent Events trial (CURE) Investigators. Effects of pretreatment with clopidogrel and aspirin followed by long-term therapy in patients undergoing percutaneous coronary intervention: the PCI-CURE study. Lancet 2001;358:527-533.

3. Hochholzer W, Trenk D, Bestehorn HP, et al. Impact of the degree of periinterventional platelet inhibition after loading with clopidogrel on early clinical outcome of elective coronary stent placement. J Am Coll Cardiol 2006;48:1742-1750

4. Bonello L, Camoin-Jau L, Arques S, et al. Adjusted clopidogrel loading doses according to vasodilator-stimulated phosphoprotein phosphorylation index decrease rate of major adverse cardiovascular events in patients with clopidogrel resistance: a multicenter randomized prospective study. J Am Coll Cardio/ 2008;51:1404-1411.

5. Angiolillo DJ, Fernandez-Ortiz A, Bernardo E, et al. Variability in individual responsiveness to clopidogrel: clinical implications, management, and future perspectives. J Am Coll Cardiol 2007;49:1505-1516.

6. Trenk D, Hochholzer W, Fromm MF, et al. Cytochrome P450 2C19 681G>A polymorphism and high on-clopidogrel platelet reactivity associated with adverse 1-year clinical outcome of elective percutaneous coronary intervention with drug-eluting or bare-metal stents. J Am Coll Cardiol 2008;51:1925-1934.

7. Giusti B, Gori AM, Marcucci R, et al. Cytochrome P450 2C19 lossof-function polymorphism, but not CYP3A4 IVS10 + 12G/A and P2Y12 T744C polymorphisms, is associated with response variability to dual antiplatelet treatment in high-risk vascular patients. Pharmacogenet Genomics 2007;17:1057-1064

8. Taubert D, von Beckerath N, Grimberg G, et al. Impact of P-glycoprotein on clopidogrel absorption. Clin Pharmacol Ther 2006;80:486-501.

9. Savi P, Herbert JM, Pflieger AM, et al. Importance of hepatic metabolism in the antiaggregating activity of the thienopyridine clopidogrel. Biochem Pharmacol 1992;44:527-532.

10. Simon T, Verstuyft C, Mary-Krause M, et al.; French Registry of Acute ST-Elevation and Non-ST-Elevation Myocardial Infarction (FAST-MI) Investigators. 
Genetic determinants of response to clopidogrel and cardiovascular events. N Engl J Med 2009;360:363-375.

11. Mega JL, Close SL, Wiviott SD, et al. Genetic variants in $A B C B 1$ and $C Y P 2 C 19$ and cardiovascular outcomes after treatment with clopidogrel and prasugrel in the TRITON-TIMI 38 trial: a pharmacogenetic analysis. Lancet 2010;376:13121319.

12. Wallentin L, James S, Storey RF, et al.; PLATO investigators. Effect of CYP2C19 and $A B C B 1$ single nucleotide polymorphisms on outcomes of treatment with ticagrelor versus clopidogrel for acute coronary syndromes: a genetic substudy of the PLATO trial. Lancet 2010;376:1320-1328.

13. Jeong YH, Tantry US, Kim IS, et al. Effect of CYP2C19*2 and *3 loss-offunction alleles on platelet reactivity and adverse clinical events in East Asian acute myocardial infarction survivors treated with clopidogrel and aspirin. Circ Cardiovasc Interv 2011;4:585-594.

14. Mega JL, Close SL, Wiviott SD, et al. Cytochrome $\mathrm{p}-450$ polymorphisms and response to clopidogrel. N Eng/ J Med 2009;360:354-362.

15. Society for Cardiovascular A, Interventions, Society of Thoracic S, et al. ACCF/ AHA Clopidogrel clinical alert: approaches to the FDA "boxed warning": a report of the American College of Cardiology Foundation Task Force on Clinical Expert Consensus Documents and the American Heart Association. Circulation 2010;122:537-557.

16. Hlatky MA, Greenland P, Arnett DK, et al.; American Heart Association Expert Panel on Subclinical Atherosclerotic Diseases and Emerging Risk Factors and the Stroke Council. Criteria for evaluation of novel markers of cardiovascular risk: a scientific statement from the American Heart Association. Circulation 2009;119:2408-2416.

17. Cook NR. Use and misuse of the receiver operating characteristic curve in risk prediction. Circulation 2007;115:928-935.

18. Pencina MJ, D'Agostino RB Sr, D'Agostino RB Jr, Vasan RS. Evaluating the added predictive ability of a new marker: from area under the ROC curve to reclassification and beyond. Stat Med 2008;27:157-72; discussion 207.

19. King SB, 3rd, Smith SC, Jr, Hirshfeld JW, Jr, et al. 2007 focused update of the ACC/AHA/SCAI 2005 guideline update for percutaneous coronary intervention: a report of the American College of Cardiology/American Heart Association Task Force on Practice Guidelines. Circulation 2008;117:261-295.

20. DeLong ER, DeLong DM, Clarke-Pearson DL. Comparing the areas under two or more correlated receiver operating characteristic curves: a nonparametric approach. Biometrics 1988;44:837-845.

21. Pencina MJ, D'Agostino RB, Pencina KM, Janssens AC, Greenland P. Interpreting incremental value of markers added to risk prediction models. Am J Epidemiol 2012; 176:473-481

22. Luo M, Li J, Xu X, Sun X, Sheng W. ABCB1 C3435T polymorphism and risk of adverse clinical events in clopidogrel treated patients: a meta-analysis. Thromb Res 2012;129:754-759.
23. Singh $M$, Shah $T$, Adigopula $S$, et al. CYP2C19*2/ABCB1-C3435T polymorphism and risk of cardiovascular events in coronary artery disease patients on clopidogrel: is clinical testing helpful? Indian Heart J 2012;64: 341-352.

24. Bergmeijer TO, Janssen PW, Schipper JC, et al. CYP2C19 genotype-guided antiplatelet therapy in ST-segment elevation myocardial infarction patientsRationale and design of the Patient Outcome after Primary PCI (POPular) Genetics study. Am Heart J 2014;168:16-22.e1.

25. Mayo Clinic. Tailored Antiplatelet Therapy Following PCI (TAILOR-PCI). ClinicalTrials.gov. http://clinicaltrials.gov/ct2/show/NCT01742117. Accessed 29 September 2015

26. Lloyd-Jones DM. Cardiovascular risk prediction: basic concepts, current status, and future directions. Circulation 2010;121:1768-1777.

27. Shuldiner AR, $\mathrm{O}^{\prime}$ Connell JR, Bliden KP, et al. Association of cytochrome P450 2C19 genotype with the antiplatelet effect and clinical efficacy of clopidogrel therapy. JAMA 2009;302:849-857.

28. Mak KH, Bhatt DL, Shao M, et al. Ethnic variation in adverse cardiovascular outcomes and bleeding complications in the Clopidogrel for High Atherothrombotic Risk and Ischemic Stabilization, Management, and Avoidance (CHARISMA) study. Am Heart J 2009;157:658-665.

29. Goto S, Toda E. Antiplatelet therapy after coronary intervention in Asia and Japan: the Asian perspective of antiplatelet intervention. Hamostaseologie 2009;29:321-325.

30. Lee SW, Park SW, Kim YH, et al. Drug-eluting stenting followed by cilostazol treatment reduces late restenosis in patients with diabetes mellitus the DECLARE-DIABETES Trial (A Randomized Comparison of Triple Antiplatelet Therapy with Dual Antiplatelet Therapy After Drug-Eluting Stent Implantation in Diabetic Patients). J Am Coll Cardiol 2008;51:1181-1187.

31. Lee SW, Park SW, Kim YH, et al.; DECLARE-LONG II Study Investigators. A randomized, double-blind, multicenter comparison study of triple antiplatelet therapy with dual antiplatelet therapy to reduce restenosis after drug-eluting stent implantation in long coronary lesions: results from the DECLARE-LONG II (Drug-Eluting Stenting Followed by Cilostazol Treatment Reduces Late Restenosis in Patients with Long Coronary Lesions) trial. J Am Coll Cardiol 2011; 57:1264-1270

32. Suh JW, Lee SP, Park KW, et al. Multicenter randomized trial evaluating the efficacy of cilostazol on ischemic vascular complications after drug-eluting stent implantation for coronary heart disease: results of the CILON-T (influence of CILostazol-based triple antiplatelet therapy ON ischemic complication after drug-eluting stenT implantation) trial. J Am Coll Cardiol 2011;57:280-289.

33. Park KW, Hwang SJ, Kwon DA, et al.; Korea Stent Thrombosis Investigators. Characteristics and predictors of drug-eluting stent thrombosis: results from the multicenter "Korea Stent Thrombosis (KoST)" registry. Circ J 2011;75: 1626-1632. 